\title{
Automatic Five-Axis CNC Feedrate Selection via Discrete Mechanistic, Geometric, and Machine Model Integration
}

\author{
Jeffrey G. Hemmett, Barry K. Fussell, Robert B. Jerard \\ Department of Mechanical Engineering, University of New Hampshire, Durham, New \\ Hampshire
}

Key words: CAD/CAM, CNC, 5-axis, Automatic Feedrate Selection, Adaptive Feedrates, Milling Simulation, Mechanistic Modeling

\begin{abstract}
The purpose of this research is to determine feasibility and develop software tools for automatically generating adaptive feedrates for use in five-axis $\mathrm{CNC}$ end milling. The complicated part geometries often involved with five-axis milling, combined with the rotational degrees of freedom of the machines, make it difficult to manually estimate acceptable feedrates without being overly conservative. Our approach for automatic feedrate generation is to use a computer simulation of the milling process. This software estimates the feeds required to maintain a desired peak cutting force on a per-tool-move basis, and consists of three distinct portions: a discrete mechanistic model, a discrete geometric model, and a model of the specific CNC machine on which the part is to be cut. The mechanistic model estimates cutting forces as a function of cut geometry, cutter-tostock relative velocity, and material constants. Used in an inverse manner, the mechanistic model may be used to estimate the feedrates necessary to maintain a constant peak cutting force. This force value may be selected to prevent cutter breakage, maintain a desired part tolerance, or to meet some other criteria (e.g. machine constraints). The results of this research have shown that it is possible to automatically generate adaptive feeds that maintain a desired force level using these combined models.
\end{abstract}

\section{INTRODUCTION}

The focus of this research is adaptive feedrate selection for five-axis contour milling of complex surfaces. The use of adaptive feedrates can reduce the machining time necessary to cut a given part, improve part tolerance, and improve the overall process reliability, as the feeds continually adjust with the changing cutting conditions. The complex surface geometries involved in contour milling, combined with the often unpredictable kinematics and controller behavior of five-axis mills, make it difficult to manually estimate acceptable feedrate values. The complexity of the problem offers little hope for efficient manual optimization. In the interest of protecting the machine and the part, manually estimated feeds are often overly conservative, based on perceived worst-case conditions which may occur very infrequently during the milling process.

Through computer simulation of the milling process it is possible to generate adaptive feeds which vary on a per tool-move basis with the changing cutting conditions, and are near optimal based on a desired force constraint. This can greatly reduce the machining time, reduce the chance for cutter breakage, and produce more predictable results. Additionally it simplifies and expedites the feed selection process.

The approach for force-based feedrate selection presented in this paper does not rely on a single model. Instead an integrated approach is used, combining three separate discrete models. These models represent the three primary contributors to the force-feedrate relation, and are referred to in this research as the mechanistic model, the geometric model, and the machine model.

The original version of this chapter was revised: The copyright line was incorrect. This has been corrected. The Erratum to this chapter is available at DOI: 10.1007/978-0-387-35392-0_40 


\section{DISCRETE MECHANISTIC MODEL OF CUTTING FORCES}

\subsection{Force Estimation}

The mechanistic model estimates cutting force vectors based on cut geometry, feedrate, and material constants. The end milling mechanistic model implemented in this research is based on Kline and DeVor's work (Kline et al., 1982 and Kline and DeVor, 1983). They use Tlusty and MacNeil's model (Tlusty and MacNeil, 1975), but instead of integrating in a closed form, they implement a numerical technique which slices the cutter into a series of discs. Using the Kline and DeVor discrete mechanistic model, the vector forces acting on the cutting tool may be estimated as

$$
\begin{aligned}
& F_{x}(\Theta)=\sum_{i=1}^{N_{z}} \sum_{k=1}^{N_{f}}\left\{-K_{R} K_{T} d z t_{c} \sin (\alpha)+K_{T} d z t_{c} \cos (\alpha)\right\} \\
& F_{y}(\Theta)=\sum_{i=1}^{N Z} \sum_{k=1}^{N_{f}}\left\{K_{R} K_{T} d z t_{c} \cos (\alpha)+K_{T} d z t_{c} \sin (\alpha)\right\}
\end{aligned}
$$

where $\quad F_{x}, F_{y}=X$ and $Y$ vector forces acting on the cutter at cutter angle $\Theta\left(l_{f}\right)$

$\Theta \quad=$ rotation angle of the cutter $(\mathrm{rad})$,

$\mathrm{N}_{\mathrm{Z}}, \mathrm{N}_{\mathrm{f}}=$ number of axial disk elements and number of flutes on cutting tool,

$\mathrm{K}_{\mathrm{R}}, \mathrm{K}_{\mathrm{T}}=$ functions of cut geometry, material constants, and cutting tool runout,

and $\mathrm{dz}=$ thickness of an axial disk element (in);

$$
\mathrm{t}_{\mathrm{c}}=\mathrm{f}_{\mathrm{t}} \sin (\alpha)
$$

where $\quad t_{c} \quad=$ chip thickness (in), assuming no cutter deflection,

$\alpha=$ angular location of current flute on current disc,

and $\mathrm{f}_{\mathrm{t}}=$ the feed-per-tooth value (in/tooth)

Essentially this model sums, over each flute on each disc of the cutting tool model, the vector force necessary to shear a chip from the stock by a flute segment which is engaged in the metal. The range of engagement is bounded by the cutter entrance and exit angles, which are denoted the 'cut geometry parameters', calculated using the geometric milling model. Feed-per-tooth is a direct function of the cutter-to-stock relative velocity, which may be related to feedrate through a machine model.

The axial discretization of the cutting tool provides a very robust model, allowing for variations in the relative velocity and cut geometry along the cutter's axis. This model also allows for the inclusion of runout (very slight eccentricity of the cutting tool), cutter tilt, and variable cutting constants in calculating the force vectors on the cutter. This model has been successfully implemented in the estimation of both three-axis and five-axis milling forces (Ersoy, 1993, Fussell et al., 1994 and Hemmett, 1994).

\subsection{Inverse Mechanistic Modeling}

The discrete mechanistic model, developed to estimate cutting force vectors acting on the cutting tool, may also be used in an inverse manner to generate feedrates necessary to maintain a constant cutting force (Fussell et al., 1995). This force may be selected based on a number of criteria, including maintaining part tolerance by limiting cutter deflection, or meeting a machine constraint such as a maximum torque value allowed on a rotary table. By selecting a desired force vector (or magnitude), the feedrate value that produces this force may be calculated with the inverse mechanistic model.

In this research an iterative approach at implementing inverse mechanistic modeling has been selected. The iterative method uses Eqs. (1), (2) and (3) along with an initial feedrate estimate, to calculate $\mathrm{X}$ and $\mathrm{Y}$ force vectors. These may be combined into a net force vector 
for comparison to the desired cutting force magnitude. If the force values do not match (within a given tolerance), the feedrate value is adjusted appropriately using a bisection or similar method, and the force value is recalculated. Typically, only a few iterations are required to match force values within a given tolerance.

\section{GEOMETRIC MODELING OF NC MACHINING}

\subsection{Stock Material and Toolpath Representation}

The geometric model performs two important functions in the feedrate selection process. It is responsible for providing the cut geometry data for the mechanistic model, and it also serves as a dynamic geometric record of the in-process workpiece. An accurate model of the in-process machining is important to ensure that correct cut geometry parameters are calculated as the simulation progresses.

Although an exact representation of the part geometry would be desirable, it is not practical to attempt to implement such an approach. Defining surfaces and calculating surface-surface intersections are computationally expensive procedures, even for relatively simple geometries. This makes a spatially continuous geometric history very difficult, if not impossible, to achieve in an efficient and robust manner using current technology.

To overcome this problem a discrete representation of the machining geometry is used. The method selected is an extended Z-buffer approach (Hemmett, 1994). This method is similar to both the ray-casting (Wang 86, Menon and Robinson, 1992, Choi and Jerard, 1998) and dexel approaches used in NC toolpath modeling and verification (Wang 1986, VanHook 1986). In this model, the workpiece is modeled as a set of discrete, parallel vectors uniformly distributed along the base of the stock, and the toolpath envelope is modeled as a set of geometric primitives (see Figure 1). This greatly simplifies calculation of the region of intersection between the cutting tool to stock, reducing it to a set of simple line-geometric primitive intersection calculations. The use of a Z-buffer model also requires only the storage of each vector's intersection location(s), simplifying geometry storage (Wang and Wang, 1986). While its lack of complexity makes the Z-buffer approach fast and robust, it is still capable of providing the required accuracy for this application.

The extended Z-buffer model is created after defining a Cartesian coordinate system denoted as the Workpiece space. The Z-buffer vectors extend in the $+Z$ direction in workpiece space, and are distributed in a grid-like pattern in the $\mathrm{X}, \mathrm{Y}$ plane. These vectors form the Z-buffer elements, and are referred to as Column Vectors (CVs) in this research. The number of CVs desired in a model is defined via a mesh size, which represents the shortest distance between adjacent $\mathrm{CV}$ s. Control over mesh size allows the user to balance computation speed and accuracy for a

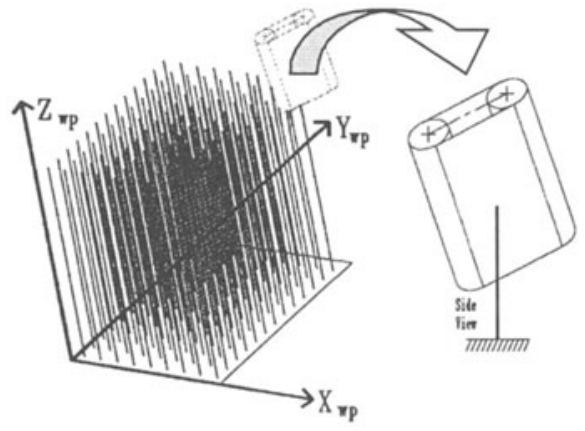

Figure 1. Geometric Model with Toolpath given application. The spatial area associated with a single vector is constant for all CVs and may be used in volume removal calculations (Ersoy, 1993).

Once the raw stock has been discretized into CVs, intersections between these vectors and a discrete approximation of the 5-axis toolpath envelope are calculated and stored (Jerard et al., 1989, Hemmett, 1994). The toolpath envelope, defined as the volume swept by the cutting 
tool between two adjacent tool positions, is modeled as a closed solid composed from a set of geometric primitives. The individual cutter movements are modelled linearly, with the cutter at a constant orientation (much like a tilted 3-axis cutter move), with all changes in cutter angular orientation occurring between each individual toolpath envelope. This simplification introduces some error, but by dividing large moves into a series of sub-moves, having smaller orientation changes, the error may be controlled. This subdivision may be defined such that the maximum error introduced by the linearly swept toolpath description is limited to an acceptable value (Quinn, 1993), minimizing the impact of the approximation.

Following the calculation of the points of intersection between the CVs and the toolpath envelope, regions along the CVs which lie within the toolpath envelope are considered "removed" (Figure 2). The Z-buffer model is referred to as "extended" as multiple intersections may be stored along a given $\mathrm{CV}$, a necessary requirement in the modeling of five-axis machining. The extended Z-buffer model deals with this by modeling each CV as a linked-list structure, allowing for the storage of multiple intersection locations ("gaps") along a given $\mathrm{CV}$, as well as simplifying intersection data management (Figure 2).

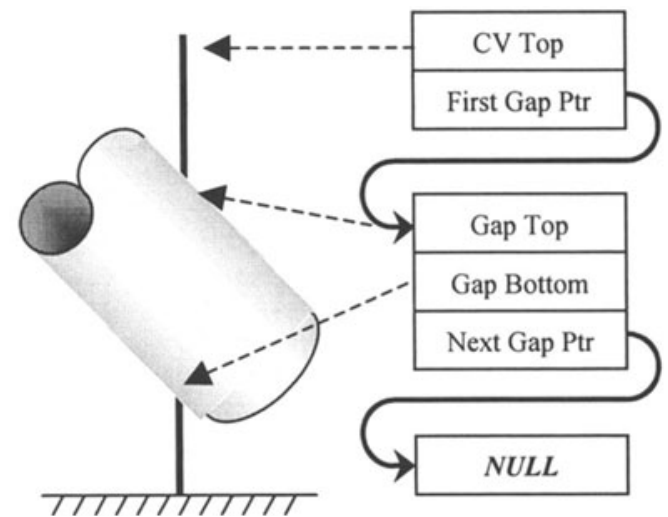

Figure 2. Multiple Intersections Between Toolpath Envelope and CVs

Conveniently, the use of an extended Z-buffer in the geometric model lends itself nicely to graphical display of shaded images, which may be viewed to verify the model. Figure 3 shows a shaded image of the in-process milling of an automotive fender.

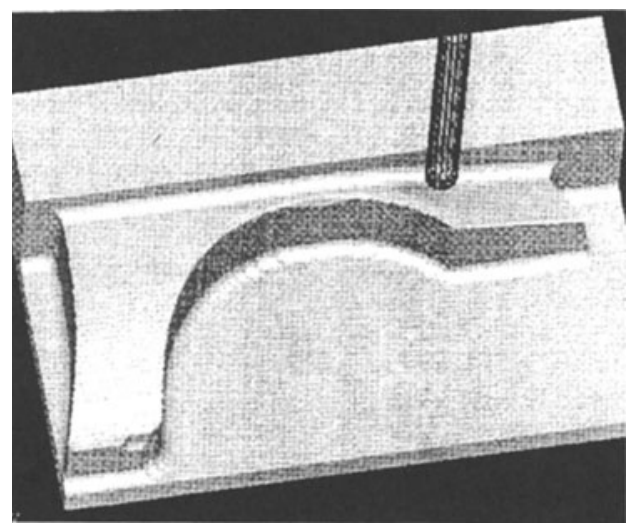

Figure 3. Shaded Image Display of In-Process Machining using Extended Z-buffer 


\subsection{Calculation of Geometric Parameters}

Following the intersection calculations for a given tool move, the cut geometry parameters are calculated. The cut geometry parameters define the region of the cutting tool engaged in the stock material for a given tool move. The force vector calculations are directly dependent upon both the magnitude and location of the contact area between the cutting tool and the workpiece.

The mechanistic model divides the cutting tool into a set of axial disc elements. For each disc there are two primary geometric cut parameter concerns. The first concern is whether or not a given axial disc element is engaged in the stock. For a disc that is engaged in the stock, the second concern for discs engaged in the stock is to define the limits of engagement, defined in the mechanistic model via a set of entrance / exit angles.

During geometric parameter calculation it is convenient to use a coordinate system that rides on the cutting tool itself; "cutting tool" coordinates. This orthogonal coordinate system has one axes in the direction of the travel vector and another vector which is orthogonal to both the direction vector and the cutter orientation vector, denoted the toolpath "Normal" vector. (see Figure 4).

The entrance / exit angles are calculated by solving for the Normal direction component of each intersection, and storing the maximum and minimum Normal positions found during a given tool move (Figure 4). Once the maximum and minimum Normal positions have been found for a move, the entrance and exit angles are found using

Where $\alpha=$ Entrance / Exit Angle value,

$$
\alpha=\sin ^{-1}(\text { NormalPos / R })+\pi / 2
$$

NormalPos $=$ Max / Min Intersection Normal Position,

$\mathrm{R}=$ Current axial disc radius,

and $\quad \pi / 2$ is added to align the angle values with mechanistic model conventions.

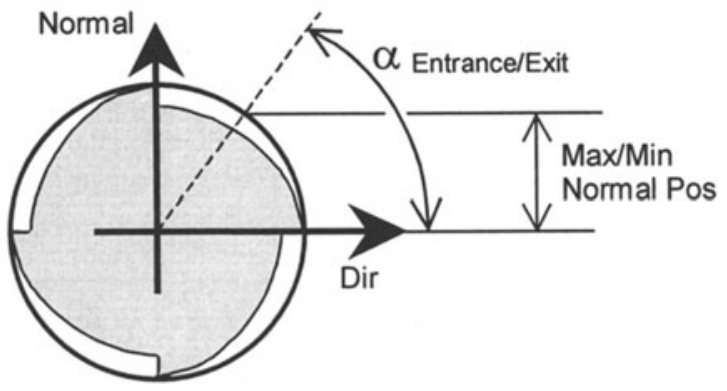

Figure 4. Entrance and Exit Angle Calculation

\section{THE CNC MACHINE MODEL}

The CNC machine model is required to relate the feedrate values entered into the CNC machine to the actual relative velocities experienced by the cutting tool relative to the stock material. This is achieved by modeling the kinematics of the machine, as well as its controller behavior, and is therefore machine specific. In order to account for the effects of the machine specific information M and G code data are used as input, as opposed to CLDAT or APT files, which lack the machine information.

The rotational degrees of freedom present in five-axis milling cause the cutter-to-stock relative velocity to vary greatly from the input feedrate value. The relative velocity value is required for the accurate calculation of chip thickness values required by the mechanistic model. Cutter-to-stock relative velocity can vary not only from one move to the next, but also 
along the length of the cutter, even for a constant input feedrate. Therefore it must be calculated not only for every tool move, but for every axial disc element of the cutting tool engaged in the stock during a given tool move.

In reality, the relative velocity varies continuously as the tool moves through space, but in our approach it is calculated once per tool move, using the value that exists at the middle of the move. Due to the small size of the tool moves (recall large moves are subdivided by the software), this results in an accurate estimation of the actual relative velocity value and has been verified through testing. The machine model may therefore also be thought of as discrete, as only a single 'sampled' instance of a continuous function is used in the calculations.

\section{MODEL INTEGRATION AND VERIFICATION}

In this research project, the inverse mechanistic, machine, and geometric models are implemented in an integrated manner. The software integration scheme is shown in Figure 5.

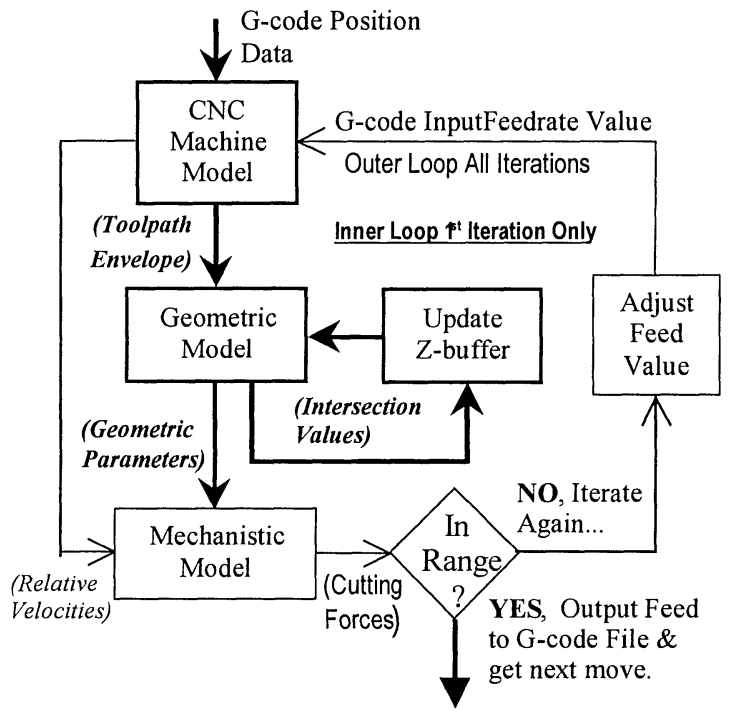

Figure 5. Flowchart of Integrated Software Model

For each tool move contained in a G-Code file, the toolpath envelope is checked for intersections with the CVs in the region of that move, and the geometric model is updated accordingly. The geometric parameters are then calculated for each intersected axial disc from the intersection data, and stored. If any intersections were found for the current tool move, the machine model is then used to calculate the relative of each axial disc element relative to the stock. Once the geometric parameters and relative velocities for all relevant axial discs have been found, the inverse mechanistic model is invoked to estimate the feedrate necessary to maintain the desired force. The inverse mechanistic model estimates the force produced by a given feedrate value, and iterates on the feed value until the estimated force is within the desired range. This procedure is repeated for each tool move in the G-Code file, resulting in a set of optimal feedrates for a given machining operation.

The initial testing which has been performed using the integrated model has produced excellent results. Currently only three-axis force data has been collected as the testing is in its preliminary stages. It is felt that if each component of the integrated model performs well, then the integrated model should too. 


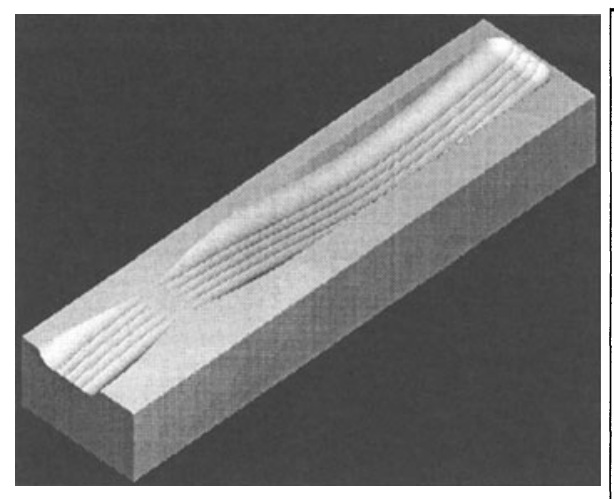

Figure 6. Rendering of Test Workpiece

Stock: 6061-T6 Aluminum $8.25 \times 6 \times 3$ inches

$\mathrm{KTC}=65,180 \mathrm{lb}_{\mathrm{f}} /$ in $^{\left(2-\mathrm{p}_{1}\right)}$

$\mathrm{K}_{\mathrm{RC}}=0.095 \mathrm{lb} \mathrm{b}_{\mathrm{f}} \mathrm{p}_{2} / \mathrm{lb}_{\mathrm{f}}$

$\mathrm{p}_{1}=0.1964 \mathrm{rad}$

$\mathrm{p}_{2}=0.22 \mathrm{rad}$

Mesh Size $=0.0075$ inches.

Cutter: 0.75 ", 4 flute HSS flat end and mill

Model disc thickness $=0.075$ in .

Coolant ON

Cuts: Desired force of $75 \mathrm{lb}_{\mathrm{f}}$

Data shown for 1st cut, (slot cut)

Table 1: Relevant Workpiece, Cutter, and Mechanistic Test Cut Parameters

Figure 6 displays the results of the geometric model portion of the milling simulation for the test workpiece. The surface milled is a sinusoid with a period of 8 " and a magnitude of 0.25 ", positioned such that the top of the curve is aligned with the top of the stock. Table 1 displays stock and cutter properties relevant to the mechanistic model.

Figure 7 displays measured force magnitudes alongside the estimated forces for the first complete pass in the generation of the sine surface. The upper set of plots shows the forces for a constant feedrate, and the lower plots for adaptive feeds generated with the integrated model. The spread in the measured values is due to cutter dynamics and runout. Note the value of interest in the measured force plots is the peak value.
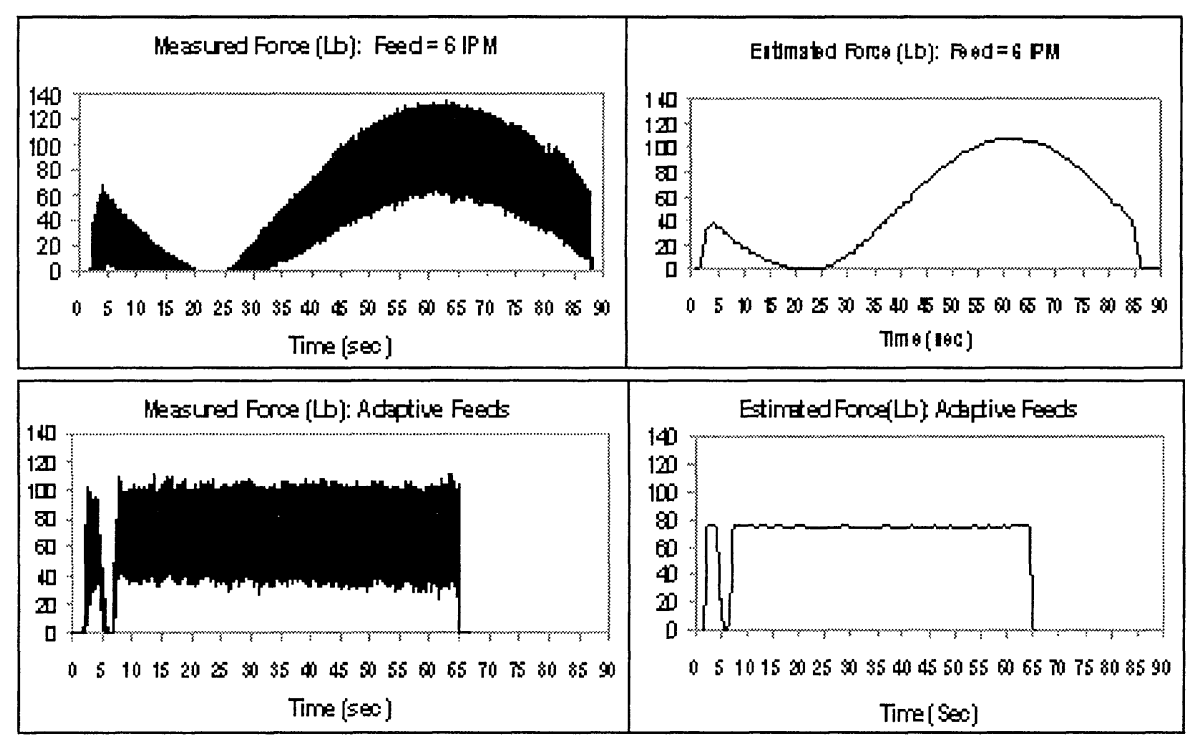

Figure 7. Measured and Estimated Force Levels for Constant Feedrate $=6$ IPM (TOP), and Adaptive Feedrate set to Maintain a Constant $75 \mathrm{lb}$. Force Magnitude (BOTTOM). 
In the upper plots a constant feedrate value of 6 IPM was selected. Note how the force signature tracks the depth of cut during constant feedrate cutting. In traditional manual feedrate selection the values are often selected based on worst case conditions, resulting in this large fluctuation in force values as the geometric cutting conditions vary between tool moves.

In the lower plots an adaptive feedrate is used, the feeds selected to maintain a constant cutting force of $75 \mathrm{Lb}$. Figure 8 shows the feedrate values used to maintain the constant force level relative to the depth of cut (depth not to scale). A maximum allowable feed value of 40 IPM was set for this test, this value is purely arbitrary and will likely increase as testing continues. In the adaptive case there is less concern with worst case conditions, as the feed adjusts to maintain the desired force for all cutting conditions. Using adaptive feeds the peak force magnitude is reduced $17 \%$ and the milling time is decreased $26 \%$ relative to the constant feed approach, providing a more conservative feedrate definition at a reduced milling time. Note that this milling time reduction does not include air time, where the tool can travel at an extremely rapid rate. Additionally, less effort is required to define the feedrate. When defining constant feeds it is often necessary to guess at a value based on the assumed worst case conditions, and then cut a test piece and adjust the feeds as necessary to achieve the desired results. In the force based feed selection approach the software performs the iterations necessary to arrive as safe feedrate values rather than the process engineer.

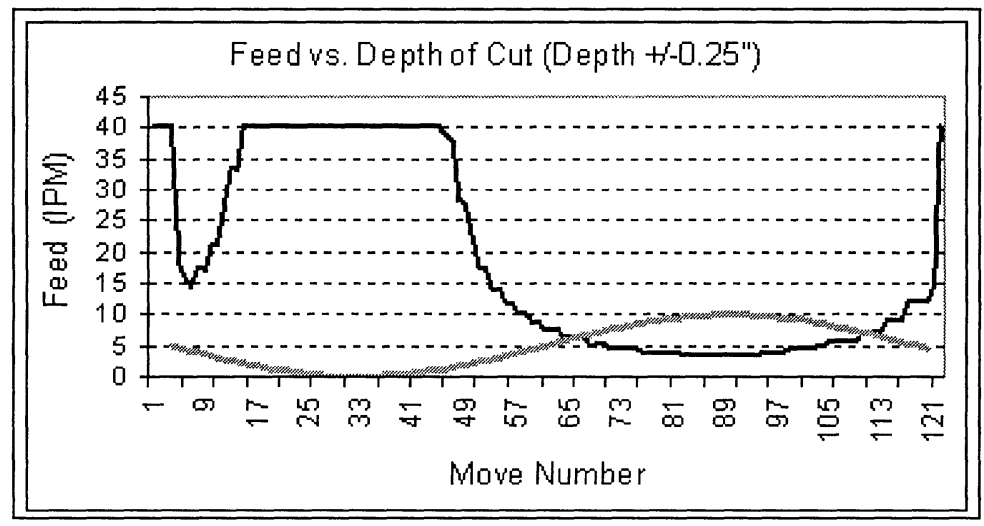

Figure 8. Adaptive Feedrate Values used in Test Cut

In both cases the shape of the force profile matches very well between the measured and estimated force values. However, the force magnitude of the measured forces exceeds the predicted values by approximately $25 \%$. This may be attributed to cutter runout, or a slight eccentricity of the cutter relative to the machine spindle. This results in a variable radius on the cutter, with an increased chip load on the side with the larger effective radii causing increased peak forces. Cutter runout also results in the large spread of the cutting forces seen in the plots due to the uneven chip load. The initial integrated model was developed without including runountl. The testing results demonstrate that runout inclusion is necessary and therefore the runout model developed by (Kline and DeVor, 1983) is being added to the model.

\section{CONCLUSIONS}

Using the discrete mechanistic model of Devor and Kline, combined with a discrete geometric milling model and a NC machine model, an integrated system capable of accurately 
estimating milling forces during the machining of sculptured surfaces has been developed. Used in an inverse manner this model is capable of selecting the feedrates necessary to maintain a desired force level. Initial testing indicates that this integrated modelling approach for three and five-axis feedrate generation is viable. Due to the inherent complications in selecting feeds for five-axis milling introduced by machine kinematics and complex cut geometry it is felt that this tool can be very useful to the manufacturing community, both to simplify process definition and to improve the milling process.

Continuing research in this area includes inclusion of cutter runout, smoothing of the feedrate data, and development of hybrid methods for feedrate estimation. Modeling the cutter runout is necessary since there will almost always be some eccentricity introduced by the tool and its holder. Acceleration limitations of the machine tool servo system dictate that no rapid variations in the feedrate be input to the NC machine. The feeds themselves should also be within the operating ability of a given machine. To meet this goal, smoothing algorithms are currently being developed which ensure that the feeds and accelerations are acceptable. Also, to reduce the computational load imposed by the feedrate selection system, methods of identifying when simpler models may be used in conjunction with the inverse mechanistic / extended Z-buffer model are being investigated, as well as performance enhancements to the existing system.

\section{REFERENCES}

1. Choi, B.K., and Jerard, R.B., 1998, Sculptured Surface Machining: Theory and Application, Kluwer Academic Publishers.

2. Ersoy, C., 1993, "Automatic Generation of Feedrates for Three-Axis End Mill Process Planning", M.S. Thesis, Dept. of Mechanical Engineering, The University of New Hampshire.

3. Fussell, B.K., Ersoy, C. and Jerard, R.B., 1992, "Computer Generated CNC Machining Feedrates", Proceedings of the Japan-USA Symposium on Flexible Automation, San Francisco, CA, pp. 377-384, 13 July.

4. Fussell, B.K., Hemmett, J.G., and Jerard, R.B., 1994, "Geometric and Mechanistic Modeling Integration for Five-Axis Milling Force Prediction", Proceedings of the Japan-USA Symposium on Flexible Automation, Kobe, Japan, pp. 747-750, 11 July.

5. Fussell, B.K., Jerard, R.B., and Durdag, O.K., 1995, "Geometric and Mechanistic Model Integration for Three-Axis CNC Feedrate Generation", ASME 1995 IMECE Joint Symposium on Concurrent Product and Process Engineering, San Francisco, CA, MED Vol. 1, pp 37-51, 12 November.

6. Jerard, R.B., Hussaini, S.Z., Drysdale, R.L., and Schaudt, B., 1989, "Approximate Methods for Simulation and Verification of Numerically Controlled Machining Programs", Visual Computer, No. 5, pp. 329-348.

7. Hemmett, J.G., 1994, "Dynamic Force Estimation and Feedrate Selection in Five Axis CNC End Milling", M.S. Thesis, Dept. of Mechanical Engineering, The University of New Hampshire.

8. Kline, W.A., and DeVor, R.E., 1983, "The effect of Runout on Cutting Geometry and Forces in End Milling", International Journal of Machine Tool Design and Research, Vol. 23, pp. 123-140, Pergamon Press.

9. Kline, W.A., DeVor, R.E., and Lindberg, J.R., 1982, "The prediction of Cutting Forces in End Milling with Application to Cornering Cuts", International Journal of Machine Tool Design and Research, Vol. 22, no. 1, pp 7-22, Pergamon Press.

10. Menon, J.P., and Robinson, D.M., 1992, "High Performance NC Verification via Massively Parallel Raycasting", Technical Report CPA92-3, The Sibley School of Mechanical and Aerospace Engineering, Cornell University.

11. Quinn, J., 1993, "Accurate Verification of Five-Axis Numerically Controlled Machining", Ph.D Thesis, Computer Science Department, Dartmouth College, Tech Report PCS-TR93-191.

12. Tlusty, J., and MacNeil, P., 1975, "Dynamics of Cutting Forces in End Milling", Annals of the CIRP, Vol 24/1.

13. VanHook, T., "Real Time Shaded Milling Display", Computer Graphics (proc. SIGGRAPH), Vol. 20, no. 4, pp15-20, Aug. 1986

14. Wang, W.P., and Wang, K.K., 1986, "Geometric Modeling for Swept Volume of Moving Solids", IEEE Computer Graphics and Applications, Vol. 6, No. 6, pp. 8-17. 